\title{
Quadrupole and Hexadecapole Correlations in Rotating Nuclei Studied within the Single- $j$ Shell Model
}

\author{
P. Magierski ${ }^{a}$, K. Burzyński ${ }^{b}$, E. Perlińska ${ }^{b}$, J. Dobaczewski $^{b}$, and W. Nazarewicz ${ }^{a-d}$ \\ ${ }^{a}$ Institute of Physics, Warsaw University of Technology \\ ul. Koszykowa 75, PL-00-662 Warsaw, Poland \\ ${ }^{b}$ Institute of Theoretical Physics, Warsaw University \\ Hoża 69, PL-00-681 Warsaw, Poland \\ ${ }^{c}$ Department of Physics and Astronomy, University of Tennessee \\ Knoxville, Tennessee 37996, U.S.A. \\ ${ }^{d}$ Physics Division, Oak Ridge National Laboratory \\ P. O. Box 2008, Oak Ridge, Tennessee 37831, U.S.A.
}

\begin{abstract}
The influence of quadrupole and hexadecapole residual interactions on rotational bands is investigated in a single- $j$ shell model. An exact shell-model diagonalization of the quadrupole-plus-hexadecapole Hamiltonian demonstrates that the hexadecapole-hexadecapole interaction can sometimes produce a staggering of energy levels in the yrast sequence; however, long and regular $\Delta I=2$ sequences are not obtained. The shell-model results are discussed in terms of the intrinsic deformations extracted by means of the self-consistent Hartree-Fock method. The angular momentum dependence of intrinsic quadrupole and hexadecapole moments $Q_{2 \mu}$ and $Q_{4 \mu}$ is investigated.
\end{abstract}

Typeset using REVTEX 


\section{INTRODUCTION}

Recently, there has been considerable interest in the behavior of hexadecapole deformations at high spins, motivated by the observation of unexpected regular variations in the spectra of superdeformed (SD) bands. Namely, in several SD bands, ${ }^{149} \mathrm{Gd}$ [1], ${ }^{153} \mathrm{Dy}$ [2], ${ }^{194} \mathrm{Hg}$ [3], ${ }^{192} \mathrm{Tl}$ [4], ${ }^{148} \mathrm{Gd}$ [5], and ${ }^{131,132} \mathrm{Ce}$ [6], a $\Delta I=2$ staggering of the dynamical moment of inertia, $\mathcal{J}^{(2)}$, has been observed. The effect is very weak and in some cases rather uncertain [7]. It manifests itself in a systematic shift of every other state in a rotational band. That is, the sequence of states with spins $I, I+4, I+8$, etc., is shifted down with respect to the sequence $I+2, I+6, I+10$, etc. In other words, the transition energies of those bands can be parametrized as:

$$
E_{\gamma}(I)=\tilde{E}_{\gamma}(I)+\epsilon(I)(-1)^{\frac{I}{2}}
$$

where $\tilde{E}_{\gamma}(I)$, the average (reference) $\gamma$-ray energy, and the perturbation $\epsilon(I)$ are smoothly varying functions of $I$. According to the data, the amplitude of the staggering, $|\epsilon(I)|$, is very small - of the order of $100 \mathrm{eV}$.

Since these oscillations separate a band into two families in which spins differ by four units of angular momentum, it seems natural to attribute their origin to a coupling between the rotational motion and hexadecapole vibrations [1,8]. This scenario requires the presence of a small component with a four-fold rotational symmetry, $\mathrm{C}_{4}$, in the mean field of the nucleus.

The effect of such a term has been investigated in phenomenological models assuming the $\mathrm{C}_{4}$ symmetry axis coinciding either with the symmetry axis (z-axis) of the quadrupole tensor [1] 9 or with the rotation axis ( $x$-axis) [1, 12, 13]. These theoretical studies have assumed the presence of a non-axial hexadecapole term in the Hamiltonian of the rotating nucleus. However, microscopic calculations based on the shell correction method [14] 17] or on the self-consistent Hartree-Fock (HF) method [18,19] predicted an extremely weak collectivity associated with the non-axial $\lambda=4$ fields.

In Ref. [20], in a simple quadrupole-plus-hexadecapole model which does not impose $a$ priori any intrinsic deformations, it has been demonstrated that the staggering in $E_{\gamma}$ may occur in certain cases. The main objective of the present study, based on the same model, is two-fold. First, we analyze the fluctuations (staggering) in the yrast line and discuss this phenomenon in terms of quadrupole and hexadecapole interactions. Second, we analyze the angular momentum dependence of intrinsic hexadecapole moments, $Q_{4 \mu}$, especially those with $\mu=2$ and 4 . To the best of our knowledge, no systematic study of this effect exists in literature. Also, experimentally, very little is known about the non-axial hexadecapole deformations, $Q_{42}$ and $Q_{44}$, and about their $I$-dependence. Some limited evidence for these exotic deformations exists from the alpha scattering studies [21, 22] around ${ }^{168} \mathrm{Er}$.

The model to test the subtle phenomena discussed above should be capable of taking into account the interplay between rotation and shape dynamics of a many-body system. In our study, we investigate a model which can be solved exactly, namely, the model of a single- $j$ shell filled with an even number of identical nucleons interacting via the multipole forces. This model, in the quadrupole-quadrupole variant [23, was widely exploited in the past to study nuclear collective effects associated with quadrupole degrees of freedom. In spite of its simplicity and a rather limited configuration space, this model is able to describe 
a variety of collective phenomena including collective rotation. For instance, even for as few as four particles in a single- $j$ shell, Mulhall and Sips [24 found rotational structures present in the spectra. Collective spectra for various particle numbers and different values of $j$ were analyzed by Friedman and Kelson [25]. Baranger and Kumar [26,27] studied quadrupole deformability and the influence of pairing, and Arima 28 discussed excited rotational structures. Recently, within the large- $j$ shell model, the quadrupole collectivity was studied by Burzyński and Dobaczewski [29].

The paper is organized as follows. The single- $j$ shell model is briefly described in Sec. II]. Section III contains the shell-model results. In particular, high-spin fluctuations in the yrast line are discussed in terms of the interplay between the quadrupole and hexadecapole components of the residual interaction. The corresponding intrinsic deformations are studied in Sec. [V within the self-consistent Hartree-Fock (HF) approach. Finally, conclusions are contained in Sec. У.

\section{THE MODEL}

The model studied in this paper is an extension of a single- $j$ shell model of Refs. 223,24. Its Hilbert space is that of a (large) single- $j$ shell occupied by identical $N$ nucleons ( $N$-even). The model Hamiltonian is rotationally invariant and takes the form:

$$
\hat{H}=\hat{H}_{\text {s.p. }}+\hat{V}_{Q Q}
$$

where $\hat{H}_{\text {s.p. }}$ is the single-particle Hamiltonian, while

$$
\hat{V}_{Q Q}=-\bar{\chi}_{2} \hat{Q}_{2} \cdot \hat{Q}_{2}-\bar{\chi}_{4} \hat{Q}_{4} \cdot \hat{Q}_{4}
$$

represents the quadrupole-quadrupole and hexadecapole-hexadecapole residual interaction. For the single- $j$ shell, $\hat{H}_{\text {s.p. }}$ reduces to a constant assumed to be zero in the following. The multipole operators appearing in Eq. (3) can be expressed as

$$
\hat{Q}_{\lambda \mu}=\sum_{m m^{\prime}}\left(j m j m^{\prime} \mid \lambda \mu\right) a_{m}^{+} \tilde{a}_{m^{\prime}}
$$

where $\tilde{a}_{m} \equiv \hat{T} a_{m} \hat{T}^{-1}$ ( $\hat{T}$ is the time reversal operator) and the dot symbols in Eq. (3) denote the scalar product

$$
\hat{Q}_{\lambda} \cdot \hat{Q}_{\lambda}=\sum_{\mu}(-)^{\mu} \hat{Q}_{\lambda \mu} \hat{Q}_{\lambda,-\mu}
$$

The Hamiltonian (2, 3) is fully described by the two constants $\bar{\chi}_{2}$ and $\bar{\chi}_{4}$ representing the strengths of the quadrupole and hexadecapole interactions, respectively. The dimension of the corresponding Hilbert space is finite. Consequently, the exact solutions of this quantum many-body problem can be obtained by means of a straightforward diagonalization [29]. This has been done in three steps, as described in the following. First, the $M$-scheme basis was constructed. It corresponds to $N$-particle wave functions $|N M n\rangle$, with $M$ being the projection of the total angular momentum $I$, and $n$ distinguishing between states with the same value of $M$. Since the model Hamiltonian is rotationally invariant, its energies do not 
depend on $M$. Hence $M$ was assumed to be equal to zero. Second, the $I$-scheme basis $|N I k\rangle$ $(M=0)$ was obtained by numerically diagonalizing the operator $\hat{I}^{2}$ ( $\hat{I}$ is the total angular momentum of the system) in the $M$-scheme basis. Here, the additional quantum number $k$ distinguishes between states with the same angular momentum $I$. Finally, the Hamiltonian has been diagonalized in the $I$-scheme basis.

For the half-filled shell there appears an additional quantum number related to the particle-hole symmetry associated with the unitary symmetry operator

$$
\hat{\mathcal{U}}=\prod_{m=-j}^{j}\left(a_{m}^{+}+a_{m}\right) .
$$

[An arbitrary but fixed order of factors in Eq. (6) is assumed.]

Since $\hat{\mathcal{U}}^{2}=(-1)^{j+1 / 2}$, depending on the value of $j, \hat{\mathcal{U}}$ is hermitian or antihermitian. By employing the identities:

$$
\hat{\mathcal{U}} a_{m}^{+}=-a_{m} \hat{\mathcal{U}}, \hat{\mathcal{U}} a_{m}=-a_{m}^{+} \hat{\mathcal{U}}
$$

it is easy to show that the particle-number operator $\hat{N}$ transforms under $\hat{\mathcal{U}}$ as

$$
\hat{\mathcal{U}}^{+} \hat{N} \hat{\mathcal{U}}=2 j+1-\hat{N}
$$

i.e., $\hat{\mathcal{U}}$ transforms a state with $N$ particles into a state with $2 j+1-N$ particles. Moreover, because of the relation

$$
\hat{\mathcal{U}}^{+} \hat{Q}_{\lambda \mu} \hat{\mathcal{U}}=-(-1)^{\mu} \hat{Q}_{\lambda,-\mu}(\lambda>0),
$$

$\hat{\mathcal{U}}$ commutes with Hamiltonian (3). Consequently, the spectra of $N$ - and $(2 j+1-N)$-particle systems are exactly degenerate, while for the half-filled shell, $N=j+1 / 2$, the eigenstates of Hamiltonian (2) acquire a new quantum number $u= \pm i^{N}$, an eigenvalue of the $\hat{\mathcal{U}}$ operator.

Properties of Hamiltonian (2) depend only on the ratio $\chi_{4}=\bar{\chi}_{4} / \bar{\chi}_{2}$. The absolute value of $\bar{\chi}_{2}$ determines the energy scale of the model. Consequently, in the following, we put $\bar{\chi}_{2}=1 \mathrm{MeV}$. The "realistic" value of $\chi_{4}$ can be estimated by means of the self-consistency relation of Refs. [30 32]. In the harmonic oscillator approximation for the average potential and assuming the nuclear radius to be $R=1.2 A^{1 / 3} \mathrm{fm}$, the self-consistent ratio $\chi_{4}$ is equal to $165 / 176$, i.e., it is very close to unity. Therefore, in our study the value of $\chi_{4}$ was varied from 0 (no hexadecapole interaction) to 1 (self-consistent limit).

The intrinsic deformations were obtained by means of the standard cranking HF procedure. The self-consistent one-body density in the rotating frame, $\hat{\rho}^{\omega}$, was found by solving the cranking $\mathrm{HF}$ equations

$$
\left[\hat{h}^{\omega}\left(\hat{\rho}^{\omega}\right), \hat{\rho}^{\omega}\right]=0
$$

where $\hat{h}^{\omega}$ is the self-consistent Routhian, $\hat{h}^{\omega}=\hat{h}-\omega \hat{I}_{x}, \hat{h}=\operatorname{Tr}\left(\hat{V}_{Q Q} \hat{\rho}^{\omega}\right)$ is the HF Hamiltonian (including the exchange term), $\hat{I}_{x}$ is the component of the total angular momentum along the $x$ axis, and $\omega$ is the rotational frequency determined from the angular momentum equation: $\operatorname{Tr}\left(\hat{\rho}^{\omega} \hat{I}_{x}\right)=I$. 
The maximum angular momentum carried out by a system of $N$ particles in a single- $j$ shell is

$$
I_{\max }=\frac{N(2 j+1-N)}{2},
$$

e.g., it is largest for a half-filled shell $\left[I_{\max }=(2 j+1)^{2} / 8\right]$. For instance, for $j=19 / 2$ and $N=10$, $I_{\max }=50$.

Having found the self-consistent density matrix, the intrinsic multipole moments,

$$
Q_{\lambda \mu}=\operatorname{Tr}\left(\hat{\rho}^{\omega} \hat{Q}_{\lambda \mu}\right), \quad(\lambda=2,4)
$$

were calculated as functions of $I$. [The multipole operators $\hat{Q}_{\lambda \mu}$ appearing in Eq. (12) are those of Eq. (蛋).] Since it is assumed that the self-consistent density matrix has three symmetry planes (i.e., $D_{2 \mathrm{~h}}$ is a self-consistent symmetry), the odd- $\mu$ components of $Q_{2}$ and $Q_{4}$ vanish, and $Q_{\lambda \mu}=Q_{\lambda-\mu}$. This is consistent with the standard definition of the intrinsic system defined in terms of the principal axis of quadrupole tensor.

It is convenient to relate the moments $Q_{\lambda \mu}(\lambda=2,4)$ to simpler deformation parameters that guarantee the unique mapping of the $Q$-surface on the parameter values. The two quadrupole moments, $Q_{20}$ and $Q_{22}$, are usually written in terms of the "polar" coordinates $\beta_{2}$ and $\gamma_{2}$ [33,34]:

$$
Q_{20}=\beta_{2} \cos \gamma_{2}, \quad Q_{22}=\frac{1}{\sqrt{2}} \beta_{2} \sin \gamma_{2} .
$$

Since in our case $\hat{\rho}^{\omega}$ has three symmetry planes, the hexadecapole tensor can be parametrized by means of the three "spherical" coordinates, $\beta_{4}, \gamma_{4}$, and $\delta_{4}$ [35]:

$$
\begin{aligned}
Q_{40} & =\beta_{4}\left(\sqrt{\frac{7}{12}} \cos \delta_{4}+\sqrt{\frac{5}{12}} \sin \delta_{4} \cos \gamma_{4}\right), \\
Q_{42} & =\frac{1}{\sqrt{2}} \beta_{4} \sin \delta_{4} \sin \gamma_{4}, \\
Q_{44} & =\beta_{4}\left(\sqrt{\frac{5}{24}} \cos \delta_{4}-\sqrt{\frac{7}{24}} \sin \delta_{4} \cos \gamma_{4}\right),
\end{aligned}
$$

where $0 \leq \delta_{4} \leq \pi$ and $-\pi \leq \gamma_{4} \leq \pi$. Since in definition (44) the radial dependence of $\hat{Q}$ has been ignored (the radial matrix element is the same for all magnetic substates, i.e., it is a scaling factor), the overall scale of the deformation variables $\beta_{2}$ and $\beta_{4}$ is given by the magnitude of the quadrupole operators (12), and is not related to the scale of the usual Bohr's shape parameters [33].

The hexadecapole shape that is axial with respect to the $z$-axis corresponds to $\gamma_{4}=\gamma_{4}^{(z)}=0$ and $\delta_{4}=\delta_{4}^{(z)}$, where $\cos \delta_{4}^{(z)}=\sqrt{7 / 12}\left(\delta_{4}^{(z)} \simeq 0.7017\right.$ or $\left.40^{\circ} 20^{\prime}\right)$. Analogously, the hexadecapole shape which is axial with respect to the $x$-axis corresponds to $\gamma_{4}=\gamma_{4}^{(x)}=2 \pi / 3$ and $\delta_{4}^{(x)}=\delta_{4}^{(z)}$. 


\section{SHELL MODEL RESULTS}

To analyze the staggering effect in collective bands, several criteria have been introduced in the literature. One possibility was suggested in Ref. [1] where the staggering was discussed in terms of

$$
\Delta^{3} E_{\gamma}(I) \equiv \frac{1}{4}\left[E_{\gamma}(I-3)-3 E_{\gamma}(I-1)+3 E_{\gamma}(I+1)-E_{\gamma}(I+3)\right],
$$

while another staggering filter,

$$
\Delta^{4} E_{\gamma}(I) \equiv \frac{3}{8}\left[E_{\gamma}(I)-\frac{1}{6}\left(4 E_{\gamma}(I-2)+4 E_{\gamma}(I+2)-E_{\gamma}(I-4)-E_{\gamma}(I+4)\right)\right],
$$

was introduced in Ref. [3]. The usefulness of quantities (15) and (16) for visualizing irregularities in rotational bands has recently been discussed in Ref. [36] where it was concluded that, in some cases, they can dramatically overemphasize perturbations in rotational levels (which occur, for instance, due to accidental degeneracies). In this context, we would like to point out that one can construct a set of quantities $\Delta^{k} E_{\gamma}$, related to various derivatives of $E_{\gamma}$ with respect to $I$. Indeed, for a function $f(x)$ defined on a discrete equidistant grid, $f_{i}=f\left(x_{i}\right)$ $\left(x_{i+1}-x_{i}=h\right)$, its derivatives can be approximated by the finite difference formulas:

$$
\begin{array}{ll}
\left(\frac{d f}{d x}\right)_{x=\left(x_{i}+x_{i+1}\right) / 2} & \approx \frac{1}{h}\left(f_{i+1}-f_{i}\right) \\
\left(\frac{d^{2} f}{d x^{2}}\right)_{x=x_{i}} & \approx \frac{1}{h^{2}}\left(f_{i-1}-2 f_{i}+f_{i+1}\right) \\
\left(\frac{d^{3} f}{d x^{3}}\right)_{x=\left(x_{i}+x_{i+1}\right) / 2} & \approx \frac{1}{h^{3}}\left(3 f_{i}-3 f_{i+1}-f_{i-1}+f_{i+2}\right) \\
\left(\frac{d f^{4}}{d x^{4}}\right)_{x=x_{i}} & \approx \frac{1}{h^{4}}\left(6 f_{i}-4 f_{i+1}-4 f_{i-1}+f_{i-2}+f_{i+2}\right) .
\end{array}
$$

It is easy to see that filters (15) and (16) can be expressed as

$$
\Delta^{3} E_{\gamma} \approx-2\left(\frac{d^{3} E_{\gamma}}{d I^{3}}\right) \quad \text { and } \quad \Delta^{4} E \approx\left(\frac{d^{4} E_{\gamma}}{d I^{4}}\right) .
$$

That is, $\Delta^{3} E_{\gamma}$ and $\Delta^{4} E_{\gamma}$ are related, respectively, to the fourth and fifth derivative of the total energy, $E$, with respect to $I$. By going to higher derivatives in defining the staggering filter, one effectively spreads out the perturbation due to configuration mixing at $I=I_{0}$ over many states around $I=I_{0}$. Consequently, in order to avoid this artificial effect, in the present study we employ the simplest filter,

$$
\Delta^{2} E_{\gamma}(I) \equiv \frac{1}{4}\left[E_{\gamma}(I-2)-2 E_{\gamma}(I)+E_{\gamma}(I+2)\right],
$$


as a measure of fluctuations [37]. According to Eq. (17a), $\Delta^{2} E_{\gamma}$ is the second derivative of $E_{\gamma}$ with respect to $I$, or

$$
\Delta^{2} E_{\gamma} \approx 2 \frac{d}{d I} \frac{1}{\mathcal{J}^{(2)}}=-2 \frac{1}{\left(\mathcal{J}^{(2)}\right)^{2}} \frac{d \mathcal{J}^{(2)}}{d I},
$$

where $\mathcal{J}^{(2)}$ is the second moment of inertia. It is worth noting that for a " $\mathrm{C}_{4}$ " spectrum, [Eq. (1)] with $\tilde{E}_{\gamma}$ linear in $I$ (perfect rotor) and $\epsilon(I)=\epsilon=$ const., all filters $\Delta^{k} E_{\gamma}(k=2,3,4)$ give results proportional to $\epsilon$.

We now proceed by applying the staggering filter (19) to the results of the exact shell model diagonalization of the single- $j$ shell Hamiltonian (2). Figure 1 displays the even- $I$ yrast lines for $N=8$ and 10 particles moving in the $j=19 / 2$ shell, and interacting with the quadrupole and hexadecapole interactions with $\chi_{4}=0,0.5$, and 1 . For the half-filled $j=19 / 2$ shell $(N=10)$ the particle-hole symmetry $\hat{\mathcal{U}}$ is preserved and the open full dots indicate states having $u=1$ and $u=-1$, respectively. The corresponding values of $\Delta^{2} E_{\gamma}$ are presented in Fig. 2.

In both cases, $N=8$ and 10 , the yrast line at $\chi_{4}=0$ and 0.5 can be understood in terms of smooth collective bands connected by large stretched $(\Delta I=2)$ transition matrix elements of the quadrupole operator, Eq. (4). In the limit of the strong hexadecapole interaction, $\chi_{4}=1$, the yrast line becomes highly perturbed and rather irregular; the quadrupole collectivity is lost, i.e., the yrast states are no longer connected by large quadrupole matrix elements.

For the half-filled shell, the yrast line obtained in the $\chi_{4}=0$ and $\chi_{4}=0.5$ variants consists of states with alternating values of $u$. Thanks to relation (9), multipole operators only connect states with different $u$ 's. Consequently, for small and intermediate values of $\chi_{4}$, the yrast line consists of long quadrupole band sequences, i.e., states connected by the $Q_{2}$ operator, while the stretched $(\Delta I=4)$ hexadecapole transitions are strictly forbidden. The situation changes dramatically for large relative strengths $\chi_{4}$. Here, due to strong hexadecapole correlations favoring the $\Delta I=4$ coupling, the alternating- $u$ pattern breaks down and the neighboring states often have the same values of $u$, i.e., they are not connected by a multipole operator. Hence, the yrast line cannot be considered as a rotational band. For the $N=8$ case, $u$ is not conserved, and the $u \rightarrow-u$ selection rule for the transition operator does not hold.

By comparing Fig. 11 with Fig. 2, one can see that the staggering filter (19) singles out all kinds of irregularities occurring along the yrast line. For example, the characteristic fluctuation at $I=16$ and $\chi_{4}=0$ (for both $N=8$ and 10) results from a small kink in the yrast line caused by a band crossing. Due to large band interaction, this irregularity is barely visible in Fig. 1.

According to Fig. 2, values of $\Delta^{2} E_{\gamma}$ increase with $\chi_{4}$. For $\chi_{4}=1$ they are an order of magnitude larger than for $\chi_{4}=0.5$. Relatively long sequences of regularly staggered points are visible for $\chi_{4}=1$ at large values of the angular momentum. One has to note, however, that the quantity $\Delta^{2} E_{\gamma}$ represents the staggering along the yrast line, which in the $\chi_{4}=1$ case does not correspond to a sequence of rotational bands. For $\chi_{4}=0.5$, the magnitude of staggering is smaller and the staggering sequences are much shorter.

As discussed above, the yrast lines calculated for $N=10$, and $\chi_{4}=0$ and $\chi_{4}=0.5$ do consist of two alternating $\Delta I=4$ sequences with the same values of $u$. However, this fact is not reflected in the corresponding staggering pattern. On the other hand, the yrast band for $\chi_{4}=1$ does not have a form of an alternating- $u$ structure, but the staggering pattern is 
clearly seen. It is, therefore, obvious that the sole existence of two different representations of states with opposite values of $(-1)^{I / 2}$ is not a sufficient condition for the appearance of the staggering pattern. In any case, the hexadecapole interactions considered in our study do not differentiate between the two particle-hole symmetries which occur for the half-filled shell.

From the shell-model results alone, one is not able to understand the origin of the longer or shorter staggering patterns obtained in the calculations. In order to analyze the underlying physics, we proceed in the next section by investigating the mean-field properties of the single- $j$ shell model.

\section{MEAN-FIELD APPROXIMATION}

The cranking HF equation (10) was solved for the Hamiltonian (2,3) and for the same values of $j, N$, and $\chi_{4}$ as used in the shell-model study. At the largest values of $\omega$, the HF solutions correspond to the fully aligned states, i.e., they are given by the Slater determinants of fermions occupying single-particle states with the largest available projections of the angular momentum on the rotation axis. This is illustrated in Fig. 3 which displays the single-particle routhians (the eigenvalues of Routhian, $\hat{h}^{\omega}|\nu\rangle=e_{\nu}^{\omega}|\nu\rangle$ ) and the corresponding single-particle angular-momentum alignments $i_{\nu}$. We see that for the large rotational frequencies, the routhians can be represented by straight lines with slopes given by constant alignments. (Recall that in the HF theory the relation $i_{\nu}=-d e_{\nu}^{\omega} / d \omega$ holds only approximately because of the $\omega$-dependence of the average HF field.)

The total alignments $\left\langle\hat{I}_{x}\right\rangle=\sum_{\nu} i_{\nu}$ (the average values of $\hat{I}_{x}$ in the many body HF states) are shown in Fig. 1 for the same parameters as used in Figs. 1 and 2. For $N=8$ and $\chi_{4}=0$ or 0.5 , the limit of a full alignment $I_{\max }=48$ is reached. A conspicuous feature of the cranking $\mathrm{HF}$ results presented in Fig. 1 is the appearance of gaps in the calculated yrast line. This can be attributed to the old problem in the description of band crossing in terms of the cranking model [38. Namely, in the region of band crossing the lowest self-consistent solution jumps as a function of $\omega$ from one continuous family of states to another.

In cases shown in Fig. 14, a band crossing occurs for $\chi_{4}=0$ and 0.5 (and for both values of $N$ ). On the other hand, for $\chi_{4}=1$ there appear many consecutive band crossings related to configuration changes, and the yrast line is composed of short pieces of continuous $\mathrm{HF}$ solutions. This effect reflects the fact discussed in Sec. III that for large values of $\chi_{4}$ the yrast states of the exact solution cannot be grouped in bands connected by strong quadrupole transition matrix elements. It also explains the strong irregularities seen in the exact yrast lines calculated with large hexadecapole interactions. When these irregularities are analyzed with a staggering filter, one may obtain sequences of staggered points; however, in the single$j$ shell model studied here this does not seem to be connected with any collective effect caused by the hexadecapole interaction. On the other hand, even an isolated band crossing, as seen in the HF results for $\chi_{4}=0$ and 0.5 , is reflected by a kink in the exact shell-model yrast line, i.e., short deviations of $\Delta^{2} E_{\gamma}$ from zero neatly exposed by the staggering filter, cf. Fig. 2.

The HF energies are compared in Fig. 1 with the results of the exact shell-model diagonalization. One can see that for $\chi_{4}=0$ the mean field approximation reproduces the exact results well [29]. Also for $\chi_{4}=0.5$ the approximation is fair, although the exact results visibly deviate at low angular momenta from a parabolic, rotor-like behavior given by the HF 
solutions. In particular, for both values of $\chi_{4}$, band crossings appear in the HF curves very close to the kinks seen in the exact yrast spectra.

On the other hand, for $\chi_{4}=1$ the HF approximation fails to reproduce the exact states at low angular momenta. Interestingly, both shell-model and HF predict the existence of yrast traps at $I \approx 16$; the multiple band crossing phenomenon is clearly correlated with the staggered shell-model energies.

At very high angular momenta, when the full alignment limit is reached, the HF results approach the exact solution. Indeed, for $I=I_{\max }$, the shell-model wave function is represented by a single, fully aligned Slater determinant, a HF state.

Based on the HF solutions, we may now discuss the nature of crossing bands. To this end, we show in Figs. 5 and 6 the average values of quadrupole and hexadecapole moments [Eq. (12)], respectively. One can see that the deformation patterns for $\chi_{4}=0$ and 0.5 are rather similar, and these patterns undergo qualitative changes when $\chi_{4}$ increases to 1 . Since our aim is to discuss collective rotation, in the following we concentrate on the case of low hexadecapole strengths.

Figure 1 shows the values of quadrupole deformation $\beta_{2}$ and $\gamma_{2}$ [Eq. (13)] for the HF yrast lines at $\chi_{4}=0.5$. It is to be noted that in our paper we consequently employ the Bohr-HillWheeler convention of $\gamma_{2}$ deformation, not the standard Lund convention. Consequently, the collective prolate rotation takes place at $\gamma_{2}=0^{\circ}$ (the $z$-axis is the symmetry axis) and $\gamma_{2}=-120^{\circ}$ (the $y$-axis is the symmetry axis), while the collective oblate rotation corresponds to $\gamma_{2}=60^{\circ}$ (the $y$-axis is the symmetry axis) or $\gamma_{2}= \pm 180^{\circ}$ (the $z$-axis is the symmetry axis). The non-collective rotation around the $x$-axis corresponds to $\gamma_{2}=-60^{\circ}$ (oblate shape) and $\gamma_{2}=120^{\circ}$ (prolate shape). The regions $\left(-60^{\circ} \leq \gamma_{2} \leq 120^{\circ}\right)$ and $\left(\gamma_{2} \leq-60^{\circ}\right.$ and $\left.\gamma_{2} \geq 120^{\circ}\right)$ are physically equivalent.

As seen in Fig. 7, the results for $N=8$ and 10 are very similar. Namely, the quadrupole deformation $\beta_{2}$ decreases with increasing angular momentum. This decrease is almost continuous across the crossing point at about $I=16$. On the other hand, at the crossing point there is a sudden change of the $\gamma_{2}$ values from about $-180^{\circ}$ (collective oblate rotation to about $-150^{\circ}$ (maximally triaxial shape with the intermediate axis being the $x$ axis).

In the fully aligned state, $I=I_{\max }$, the equilibrium shape of a system with $N<j+1 / 2$ corresponds to $\gamma_{2}=\gamma_{2}^{(x)}=-60^{\circ}$. This is so because the eigenstates of $I_{x}$ cannot have nonzero average values of $\hat{Q}_{2, \mu_{x}=2}$, i.e., the $x$ axis must be the symmetry axis, and, moreover, for $N<j+1 / 2$ the shape must be oblate. In this limit, values of quadrupole moments along the $z$ axis are proportional to the $Q_{20 x}$ values calculated along the $x$ axis. Namely: $Q_{20}=-\frac{1}{2} Q_{20 x}$, $Q_{22}=\frac{1}{2} \sqrt{\frac{3}{2}} Q_{20 x}$, and $\beta_{2}=\left|Q_{20 x}\right|$. This is seen in Fig. 7 for $N=8$ at the high-angularmomentum end of the band. On the other hand, for the half-filled shell the aligned state has $\beta_{2}=0$, and this point is approached along the $\gamma_{2}=-150^{\circ}$ line.

Of course, this description in terms of the shape characteristics pertains only to the average values of the quadrupole moments, $Q_{20}$ and $Q_{22}$, which have well-known values for ellipsoidal shapes. In the following, we refer to the bands below and above the crossing as the collective oblate and triaxial bands, respectively, and the band crossing can be interpreted as a sudden transition caused by a change in the shape of the system.

We may now discuss the evolution of the hexadecapole shape of the system within the 
oblate and triaxial bands. The oblate bands do not exhibit any tangible non-axial hexadecapole deformations, i.e., they have $Q_{42} \approx Q_{44} \approx 0$. On the other hand, in the triaxial bands all three components of the hexadecapole tensor are nonzero. In both bands the hexadecapole deformations are fairly similar for $\chi_{4}=0$ and 0.5 , i.e., they weakly depend on the fact whether the hexadecapole interaction has or has not been taken into account. This suggests a simple interpretation of the hexadecapole moments as being induced by the quadrupole deformations. Indeed, ellipsoidal shapes have nonvanishing hexadecapole moments which can be expressed through quadrupole moments or deformations. This leads to rather stringent conditions for the hexadecapole moments [39 41].

The assumption of the strong coupling of $\lambda=4$ deformations to the intrinsic frame defined through quadrupole deformations requires that the hexadecapole tensor depends on products of quadrupole tensors. This leaves a freedom of many possible parametrizations 41] defined through three scalar functions $h_{2}, h_{3}$, and $h_{4}$, depending on $\beta_{2}$ and $\cos 3 \gamma_{2}$. In the case that only $h_{3}$ is different from zero, one obtains:

$$
\begin{gathered}
\tilde{\beta}_{4}=\left|h_{3}\right| \sqrt{\left(\frac{5}{12}+\frac{7}{12} \cos ^{2} 3 \gamma_{2}\right)}, \\
\cos \tilde{\delta}_{4}= \pm \frac{\cos 3 \gamma_{2}}{\sqrt{\frac{5}{7}+\cos ^{2} 3 \gamma_{2}}}, \\
\cos \tilde{\gamma}_{4}= \pm \cos \gamma_{2}, \quad \sin \tilde{\gamma}_{4}= \pm \sin \gamma_{2} .
\end{gathered}
$$

[There are two possible sign choices in Eqs. (21b) and (210): either $\tilde{\gamma}_{4}=\gamma_{2}$ or $\tilde{\gamma}_{4}=\gamma_{2} \pm \pi$.]

Expressions (21) define shapes for which the hexadecapole moments follow the quadrupole moments and do not constitute any independent dynamical deformations. (For the general parametrization for the hexadecapole tensor, see Ref. 41].) According to Eq. (21a) the value of $\left|h_{3}\right|$ can always be chosen in such a way that the strong-coupling and selfconsistent values of $\beta_{4}$ are equal, i.e., $\beta_{4}=\tilde{\beta}_{4}$. On the other hand, the strong-coupling values of angles $\tilde{\delta}_{4}$ and $\tilde{\gamma}_{4}$ depend solely on $\gamma_{2}$.

It turns out that the HF results obtained for low and intermediate values of $\chi_{4}$ can be understood in terms of the strong-coupling limit given by Eq. (21). Figure 8 shows the calculated HF hexadecapole deformations $\beta_{4}, \delta_{4}$, and $\gamma_{4}$ together with the strong-coupling values of $\tilde{\delta}_{4}$ and $\tilde{\gamma}_{4}$ given by Eqs. (21b) and (21c), respectively. On can see that the assumption of a strong coupling accounts very well for the complicated $I$-dependence of $\delta_{4}$ and $\gamma_{4}$ presented in Fig. 6. Moreover one should notice that for the case of $N=8$ the values of $\delta_{4}$ and $\gamma_{4}$ are approaching the limit of the fully aligned state along $x$-axis where only the value of $\hat{Q}_{40 x}$ remains nonzero.

It is interesting to note that in the $N=10$ variant and $I=50$ (termination point), hexadecapole deformation $\beta_{4}$ vanishes for $\chi_{4}=0.5$. Indeed, in the limit of angular momentum alignment, the expectation value $\left\langle j m_{x}\left|Q_{\lambda \mu}\right| j m_{x}\right\rangle$ is proportional to $d_{\mu 0}^{\lambda}\left(\frac{\pi}{2}\right)\left\langle j m_{x} \lambda 0 \mid j m_{x}\right\rangle$, i.e., it depends only on $m_{x}^{2}$ for even values of $\lambda\left[\left\langle j m_{x} \lambda 0 \mid j m_{x}\right\rangle \approx P_{\lambda}\left(\frac{m_{x}}{j}\right)\right.$ and for large values of $j$ ]. Hence the $\mathrm{HF}$ expectation values $Q_{4 \mu}$ vanish in the fully aligned state of the half-filled shell $\left(\int_{1}^{0} P_{\lambda}(x) d x=0\right.$ for even values of $\left.\lambda\right)$.

In the case of $N=8$ particles, the average values of $\hat{Q}_{4 \mu}$ can be expressed by the axial hexadecapole moment calculated along the $x$ axis. Namely, the following relations hold: 
$Q_{40}=\frac{3}{8} Q_{40 x}, Q_{42}=-\frac{1}{8} \sqrt{10} Q_{40 x}, Q_{44}=\frac{1}{8} \sqrt{\frac{35}{2}} Q_{40 x}$, and $\beta_{4}=\left|Q_{40 x}\right|$. Consequently, for $I=48$ the parameters $\delta_{4}$ and $\gamma_{4}$ are approaching the values of $\delta_{4}^{(x)}$ and $\gamma_{4}^{(x)}$, respectively.

We conclude this section by presenting in Fig. 9 the hexadecapole deformation $\beta_{4}$ for $N=8$ and 10 as a function of the coupling constant $\chi_{4}$ at $I=40$, i.e., for the triaxial bands. On can see that for both particle numbers, $\beta_{4}$ steadily increases with $\chi_{4}$ in the whole range of $\chi_{4}$. That is, the hexadecapole phase transition that would manifest itself in a rapid local increase of $\beta_{4}$ with $\chi_{4}$, is not present in the single- $j$ shell model.

\section{CONCLUSIONS}

In the present paper we studied the single- $j$ shell model describing identical particles interacting via the quadrupole and hexadecapole residual interaction. The model was solved exactly, and the resulting yrast structures obtained for different strengths of the hexadecapole interaction were analyzed. The model was also solved within the Hartree-Fock approximation, where the results could be interpreted in terms of the standard shape variables. Results of the calculations were presented for the $j=19 / 2$ shell and particle numbers $N=8$ and 10. Calculations have also been performed for other values of $j$ and $N$, but the main conclusions can be drawn from the restricted set of results presented in the paper.

The purpose of our study was two-fold. First, we analyzed the conjecture that the hexadecapole $\lambda=4$ degree of freedom might be responsible for the $\Delta I=2$ staggering effect in rotational bands. Second, we investigated the evolution of the hexadecapole shapes with angular momentum.

Our study shows that by including the hexadecapole interaction in the single- $j$ model, one can sometimes obtain a staggering of yrast energies. However, a long and regular sequence of $\Delta I=2$ staggered energies cannot be obtained. This negative result suggests that the experimental data probably cannot be explained in terms of the coupling between rotation and hexadecapole vibrations.

For a relatively weak strength of the hexadecapole interaction, one obtains regular collective quadrupole bands which do not exhibit any staggering. One also systematically obtains the effect of a crossing between two different shape configurations: an oblate-shape collective band at low angular momenta and a triaxial band at high angular momenta. The irregularities which are present in the yrast line in the crossing region should not be confused with the staggering phenomenon. As discussed in Ref. [36], such a misinterpretation may easily happen especially when a multi-point staggering filter is used to extract the staggering amplitude from the calculated energies.

For a relatively strong hexadecapole interaction, the yrast line becomes rather irregular and the states are no longer connected by strong quadrupole transition matrix elements. In the mean-field picture, such a yrast structure can be interpreted as composed of several different bands crossing one another. In some cases discussed in this paper, such multiple crossings can give rise to staggering patterns.

In the studied model, one obtains nonzero hexadecapole moments even without including the hexadecapole interaction. These moments are rather weakly affected by the hexadecapole interaction. The hexadecapole deformations calculated in the single- $j$ model simply 
follow the quadrupole deformations, i.e., they can be discussed as resulting from a strong coupling between the $\lambda=2$ and $\lambda=4$ modes. Within the collective oblate bands, the hexadecapole moment is axial and oriented along the symmetry axis of the quadrupole deformation. For triaxial bands, the hexadecapole deformation also becomes triaxial, i.e., all three components of the hexadecapole moment differ from zero. They can be nicely interpreted within the strong coupling assumption, and depend in a simple geometrical way on the quadrupole asymmetry angle $\gamma_{2}$. It is worth noting that our self-consistent results strongly prefer one particular parametrization of the hexadecapole tensor of Ref. [41], namely the strong-coupling expression with $h_{2}=h_{4}=0$.

\section{ACKNOWLEDGMENTS}

This research was supported in part by the Polish Committee for Scientific Research under Contracts Nos. 2P03B 09809 and 2 P03B 034 08, by a computational grant from the

Interdisciplinary Centre for Mathematical and Computational Modeling (ICM) of Warsaw University, and by the U.S. Department of Energy (DOE) through Contract No. DE-FG0296ER40963 with the University of Tennessee. Oak Ridge National Laboratory is managed for the U.S. DOE by Lockheed Martin Energy Research Corp. under Contract No. DEAC05-96OR22464. 


\section{REFERENCES}

[1] S. Flibotte, et al., Phys. Rev. Lett. 71, 4299 (1993).

[2] B. Cederwall, et al., Phys. Lett. B346, 244 (1995).

[3] B. Cederwall, et al., Phys. Rev. Lett. 72, 3150 (1994).

[4] S.M. Fischer et al., Phys. Rev. C53, 2126 (1996).

[5] G. de Angelis et al., Phys. Rev. C53, 679 (1996).

[6] A.T. Semple et al., Phys. Rev. Lett. 76, 3671 (1996).

[7] R. Krücken et al., Abstract, Int. Conf. on Nuclear Structure at the Limits, Argonne, Illinois, 1996, ANL/PHY-96/1, p. 21.

[8] L.K. Peker, S. Pearlstein, J.O. Rasmussen, and J.H. Hamilton, Phys. Rev. Lett. 50, 1749 (1983).

[9] I. Hamamoto and B. Mottelson, Phys. Lett. B333, 294 (1994).

[10] P. Magierski, K. Burzyński, J. Dobaczewski, and W. Nazarewicz, Acta Phys. Pol. B26, 291 (1995).

[11] A.O. Macchiavelli et al., Phys. Rev. C51, R1 (1995).

[12] I.M. Pavlichenkov and S. Flibotte, Phys. Rev. C51, R460 (1995).

[13] P. Magierski, Acta Phys. Pol. B27, 127 (1996).

[14] I. Ragnarsson, private communication, 1994.

[15] S. Frauendorf, J. Meng, and J. Reif, Proc. Int. Conf. on Physics from Large $\gamma$-ray Detector Arrays, Berkeley, 1994, Volume 2, LBL-35687, p. 52.

[16] F. Dönau, S. Frauendorf, and J. Meng, Phys. Lett. B, in press.

[17] W.D. Luo, A. Bouguettoucha, J. Dobaczewski, J. Dudek, and X. Li, Phys. Rev. C52, 2989 (1995).

[18] P. Magierski, P.-H. Heenen, and W. Nazarewicz, Phys. Rev. C51, R2880 (1995).

[19] A. Bouguettoucha, J. Dobaczewski, J. Dudek, W. D. Luo, and H. Molique, to be published.

[20] K. Burzyński, P. Magierski, J. Dobaczewski, and W. Nazarewicz, Phys. Scr. T56, 228 (1995).

[21] I.M. Govil, H.W. Fulbright, D. Cline, E. Wesołowski, B. Kotliński, A. Backlin, and K. Gridnev, Phys. Rev. C33, 793 (1986).

[22] I.M. Govil, H.W. Fulbright, and D. Cline, Phys. Rev. C36, 1442 (1987).

[23] S.T. Belyaev, Mat. Fys. Medd. Dan. Vid. Selsk. 31 (No. 11) (1959).

[24] W.J. Mulhall and L. Sips, Nucl. Phys. 57, 565 (1964).

[25] M.I. Friedman and I. Kelson, Nucl. Phys. A144, 209 (1970).

[26] M. Baranger and K. Kumar, Nucl. Phys. 62, 113 (1965).

[27] M. Baranger and K. Kumar, Nucl. Phys. A110, 490, 529 (1968); A122, 244 (1968).

[28] A. Arima, Prog. Theor. Phys., Suppl. extra number, 489 (1968).

[29] K. Burzyński and J. Dobaczewski, Phys. Rev. C51, 1825 (1995).

[30] D.J. Rowe, Phys. Rev. 162, 866 (1967).

[31] A. Bohr and B.R. Mottelson, Nuclear Structure, vol. 2 (W.A. Benjamin, New York, 1975).

[32] H. Sakamoto and T. Kishimoto, Nucl. Phys. A501, 205 (1989).

[33] A. Bohr, Mat. Fys. Medd. Dan. Vid. Selsk. 26, No. 14 (1952).

[34] D.L. Hill and J.A. Wheeler, Phys. Rev. 89, 1102 (1953).

[35] S.G. Rohoziński and A. Sobiczewski, Acta. Phys. Pol. B12, 1001 (1981). 
[36] V. Reviol, H.-Q Jin, and L.L. Riedinger, Phys. Lett. B371, 19 (1996).

[37] C. Baktash, private communication, 1995.

[38] I. Hamamoto, Nucl. Phys. A271, 15 (1976); R.A. Sorensen, Rev. Mod. Phys. 45, 353 (1973).

[39] S.G. Rohoziński, Report INR 1520/VII/PH/B, Warszawa 1974, unpublished.

[40] W. Nazarewicz and P. Rozmej, Nucl. Phys. A369, 396 (1981).

[41] S.G. Rohoziński, to be published. 


\section{FIGURES}

FIG. 1. The exact yrast spectrum (circles) of the quadrupole-plus-hexadecapole model for the $j=19 / 2$ shell filled with $N=8$ (left) and 10 (right) particles, and $\chi_{4}=0$ (top), 0.5 (middle), and 1 (bottom). The yrast bands calculated in the HF method are indicated by solid lines. For $N=10$, open and full circles correspond to the values $u=1$ and $u=-1$, respectively, of the particle-hole symmetry quantum number.

FIG. 2. Staggering parameter $\Delta^{2} E_{\gamma}$ [Eq. (19)] as a function of $I$ along the yrast lines obtained in the shell-model results presented in Fig. 1.

FIG. 3. Top: Self-consistent single-particle routhians, $e_{\nu}^{\omega}$, for $j=19 / 2$ and $N=10$ (yrast line) as functions of the rotational frequency $\omega$. Bottom: the corresponding single-particle alignments. Solid lines: signature $r=+i$. Dashed lines: $r=-i$.

FIG. 4. Angular momentum (the expectation value of $\hat{I}_{x}$ in the cranking HF state) as a function of $\omega$ for the $j=19 / 2$ shell filled with $N=8$ (left) and 10 (right) particles, and $\chi_{4}=0$ (top), 0.5 (middle), and 1 (bottom).

FIG. 5. Equilibrium quadrupole moments $Q_{20}$ (solid line) and $Q_{22}$ (dot-dashed line) for HF yrast solutions as functions of angular momentum for the $j=19 / 2$ shell with $N=8$ (left) and 10 (right) particles, and $\chi_{4}=0$ (top), 0.5 (middle), and 1 (bottom).

FIG. 6. Same as in Fig. 5 except for equilibrium hexadecapole moments $Q_{40}$ (solid line) and $Q_{42}$ (dot-dashed line), and $Q_{44}$ (dotted line).

FIG. 7. Equilibrium deformations $\beta_{2}$ and $\gamma_{2}$ [Eq. (13)] as functions of angular momentum for the HF yrast states obtained in the $j=19 / 2$ shell filled with $N=8$ (solid line) or $N=10$ (dot-dashed line) particles, and for $\chi_{4}=0.5$. The limit of axial quadrupole shapes are indicated by dotted lines $\left(\gamma_{2}^{(x)}\right.$ : axial symmetry with respect to $x$-axis; $\gamma_{2}^{(z)}$ : axial symmetry with respect to $z$-axis).

FIG. 8. Equilibrium hexadecapole deformations $\beta_{4}, \delta_{4}$, and $\gamma_{4}$ [Eq. (14)] as functions of angular momentum for $j=19 / 2, \chi_{4}=0.5$, and $N=8$ (left) and 10 (right). Values of $\tilde{\delta}_{4}$ and $\tilde{\gamma}_{4}$ obtained in the strong-coupling limit [Eq. (21)] are denoted by dashed lines. The limits of axial hexadecapole

shapes are indicated by dotted lines $\left(\delta_{4}^{(x)}, \gamma_{4}^{(x)}\right.$ : axial symmetry with respect to $x$-axis; $\delta_{4}^{(z)}, \gamma_{4}^{(z)}$ : axial symmetry with respect to $z$-axis).

FIG. 9. Equilibrium hexadecapole deformation $\beta_{4}$ as a function of $\chi_{4}$ for the HF yrast states at a fixed angular momentum, $I=40$. Calculations were performed for the $j=19 / 2$ shell filled with $N=8$ (solid line) or $N=10$ (dashed line) particles. 


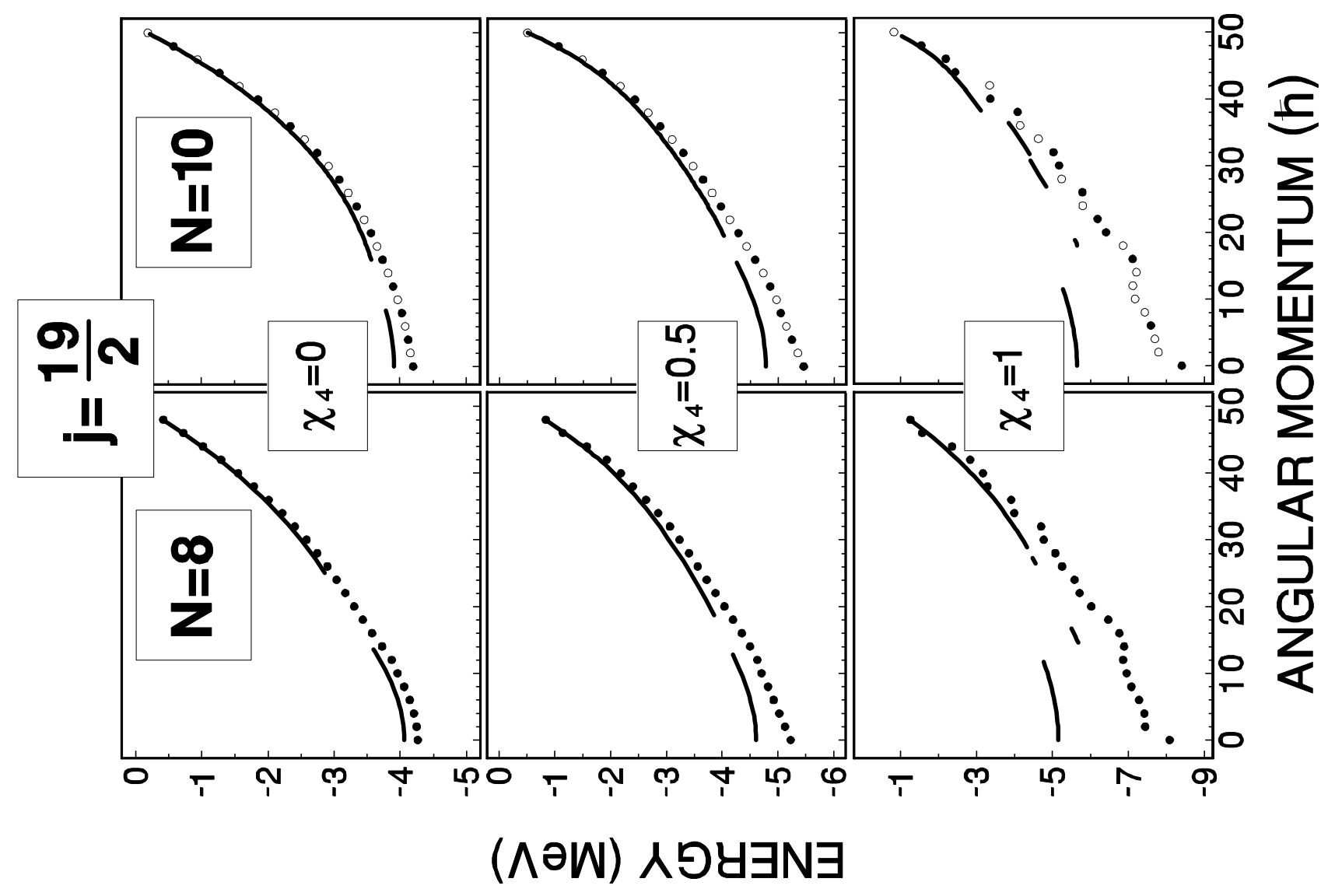




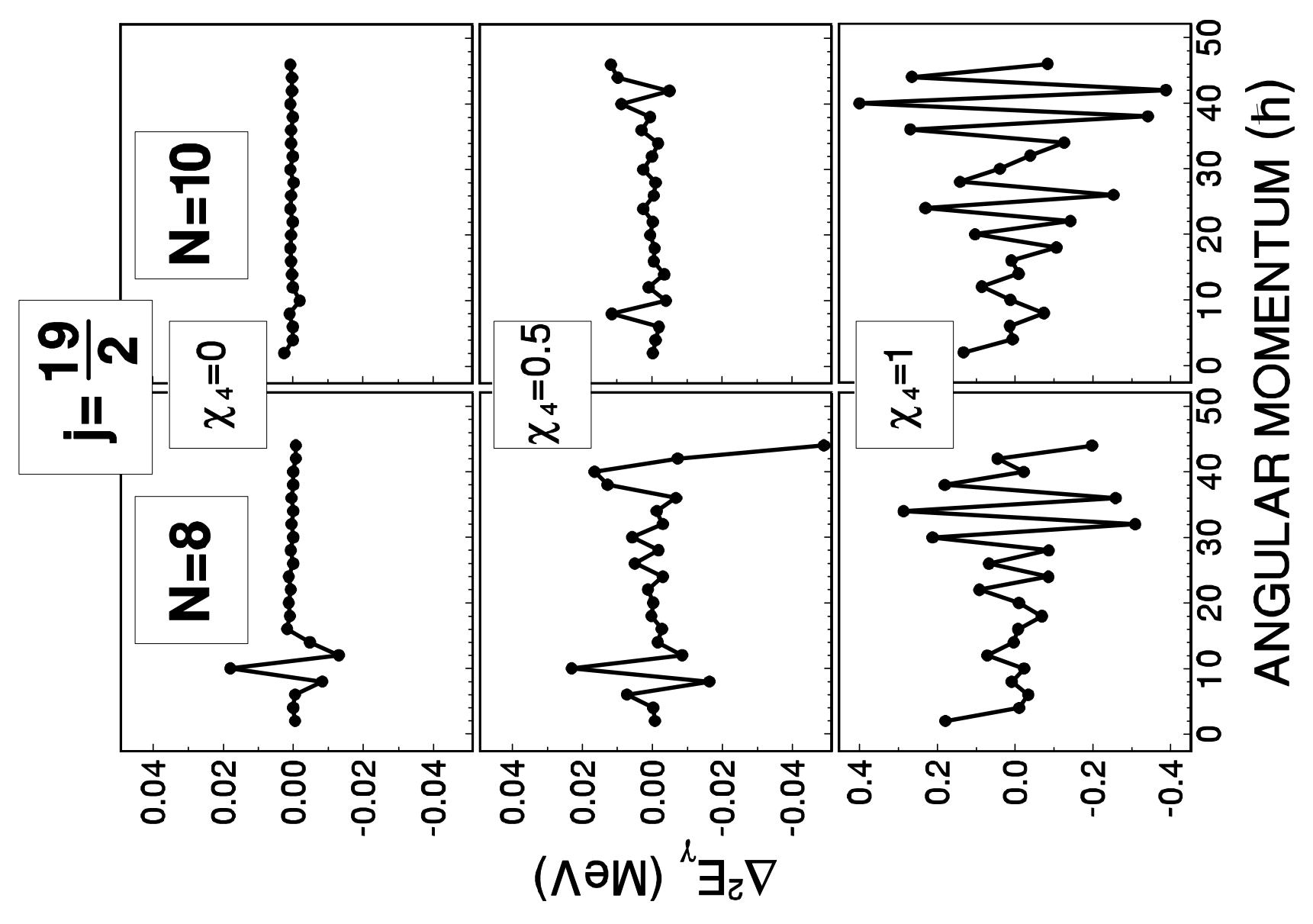




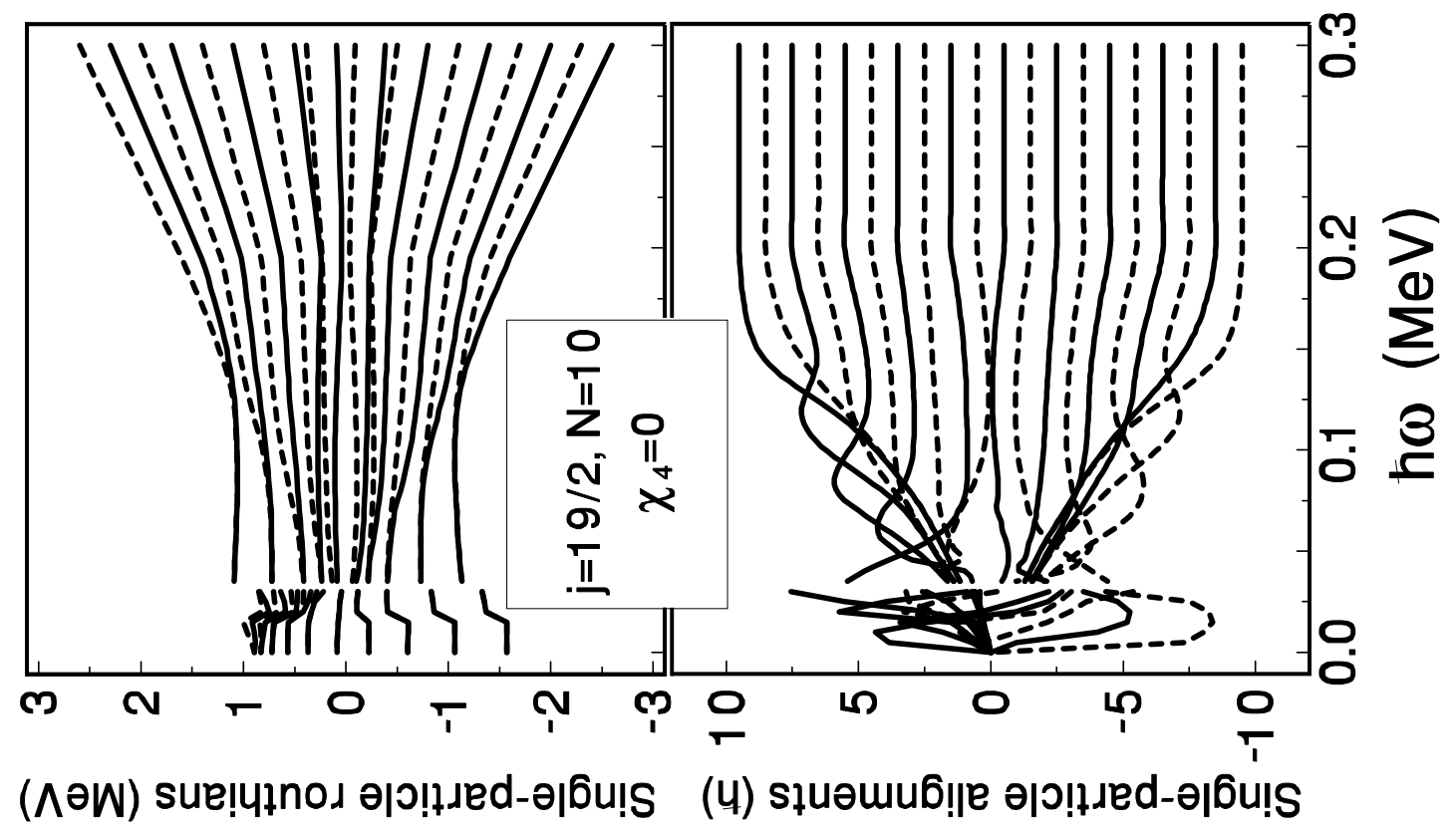




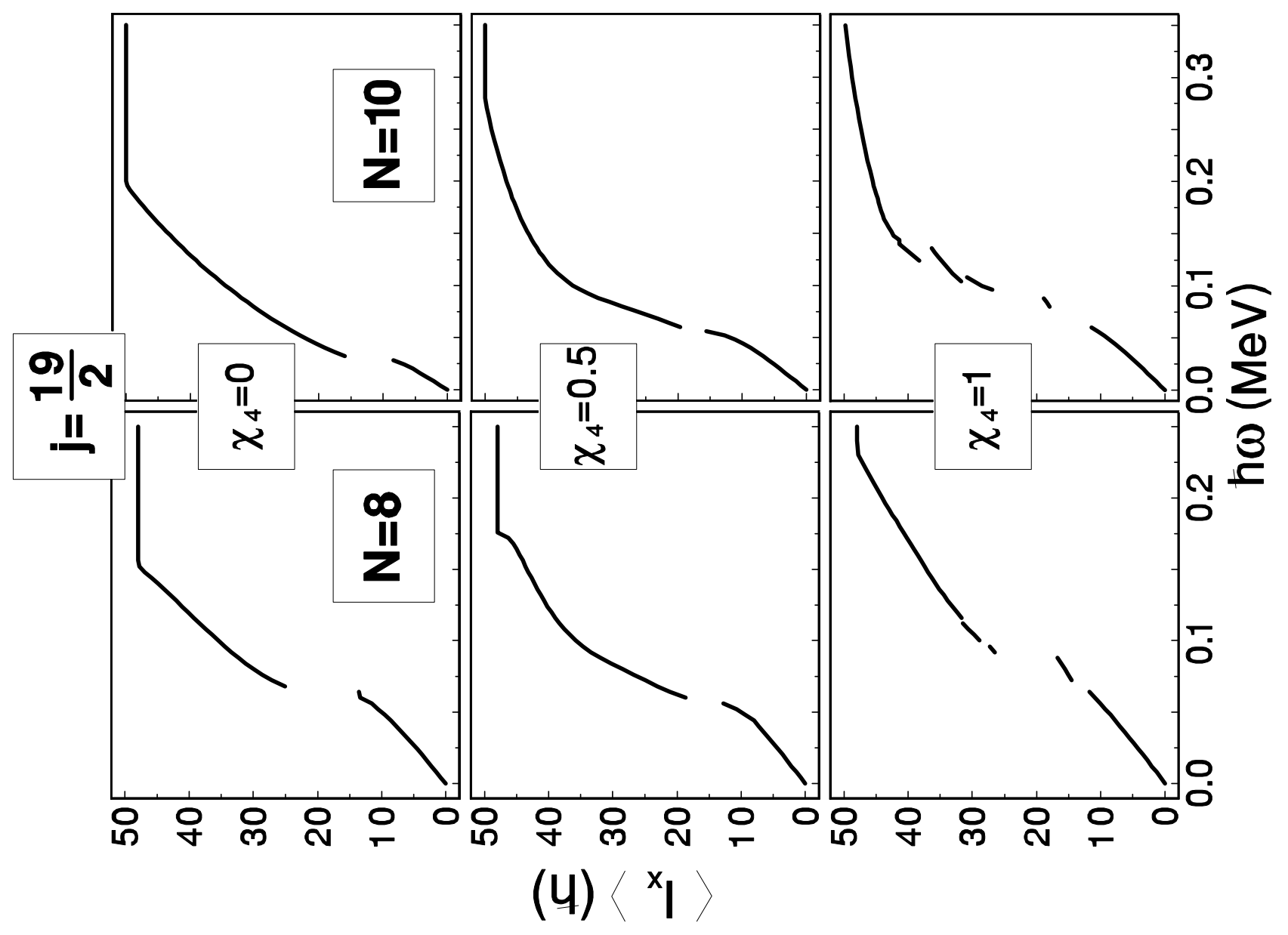




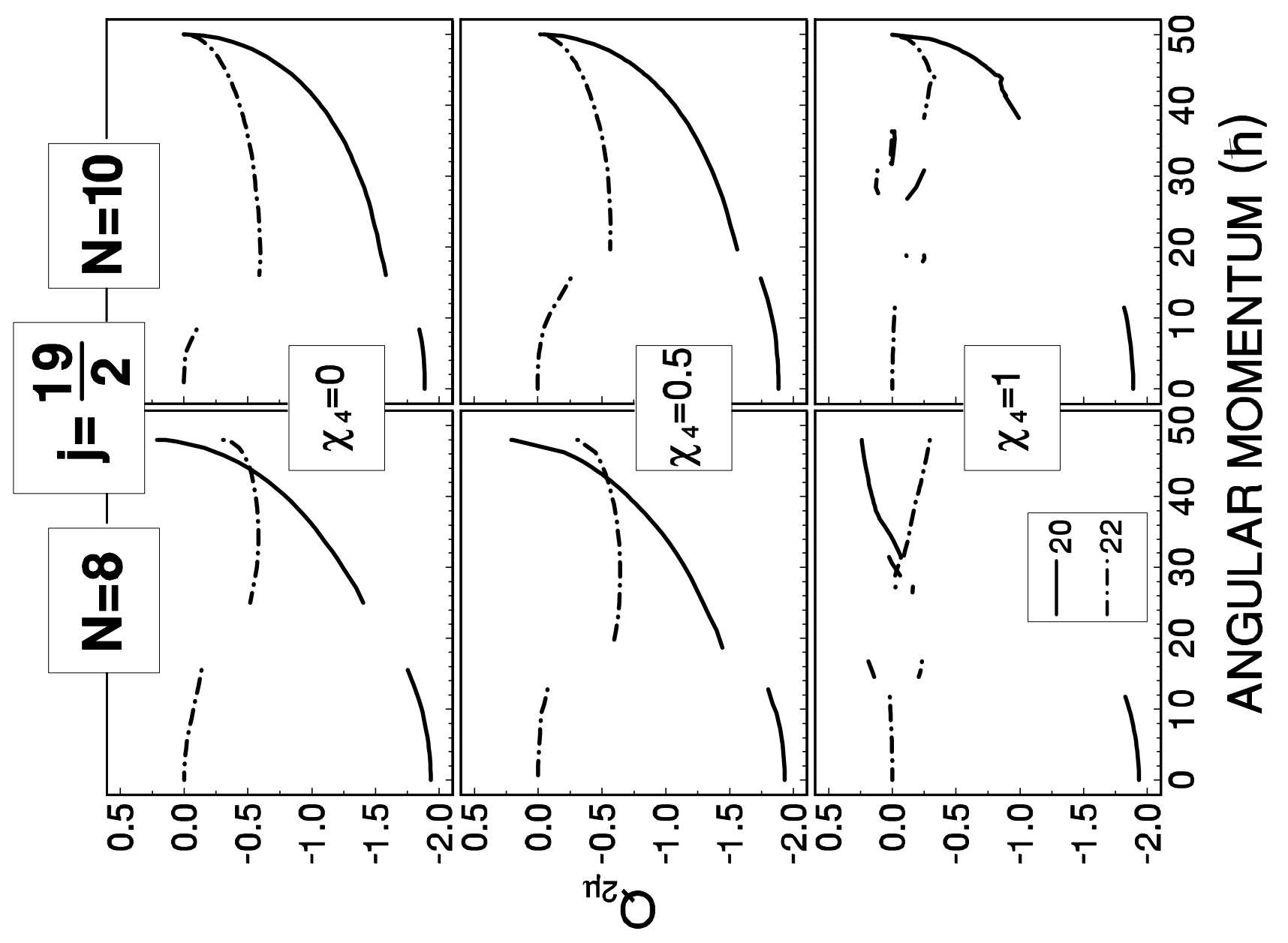




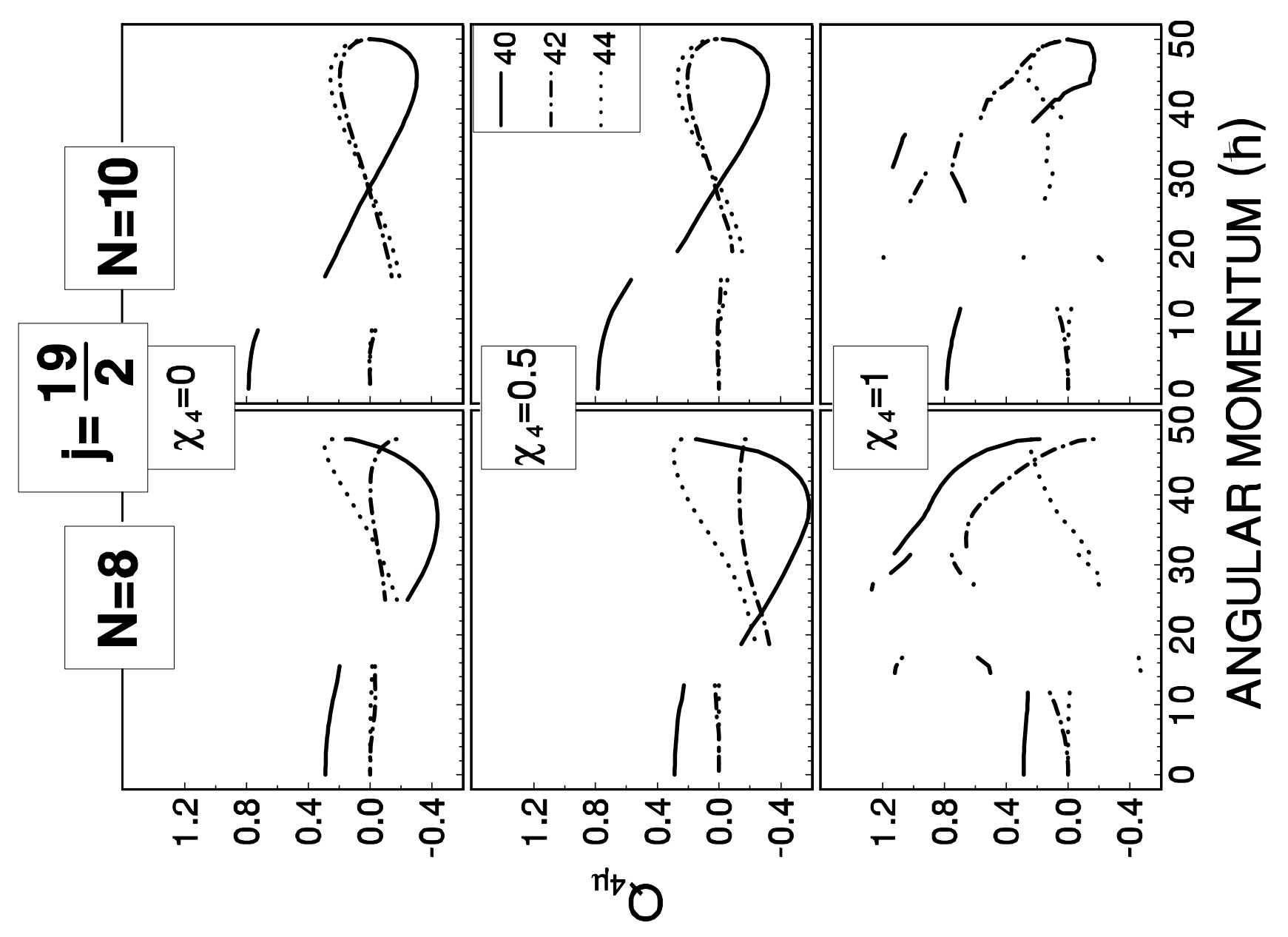




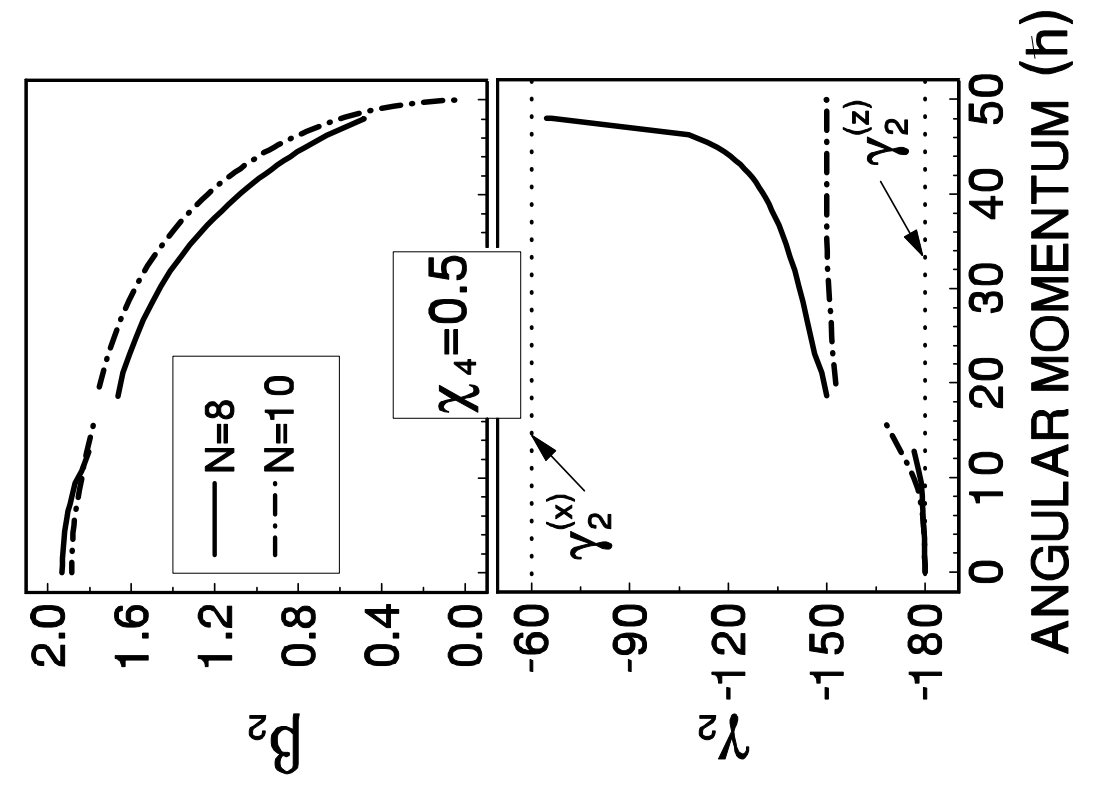




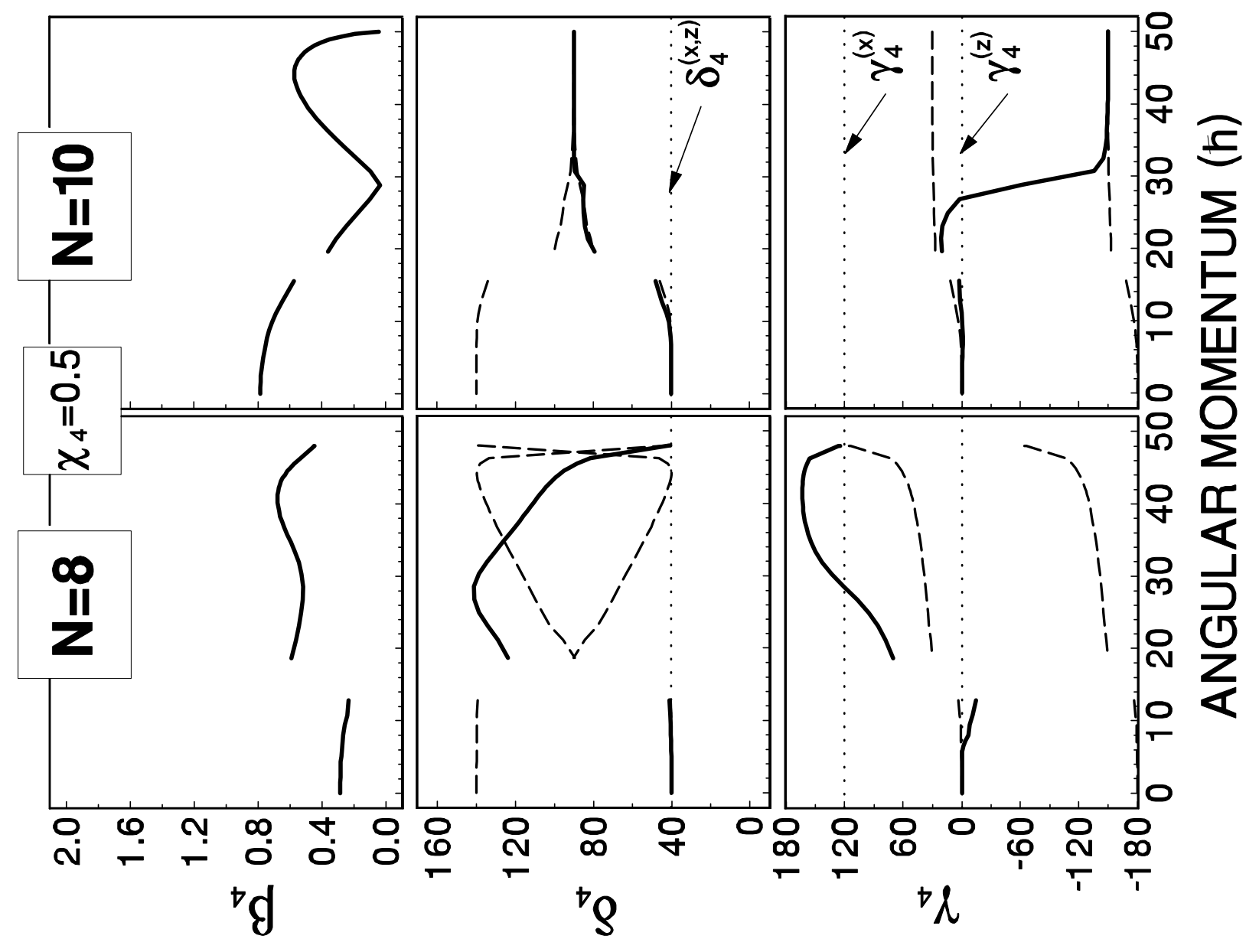




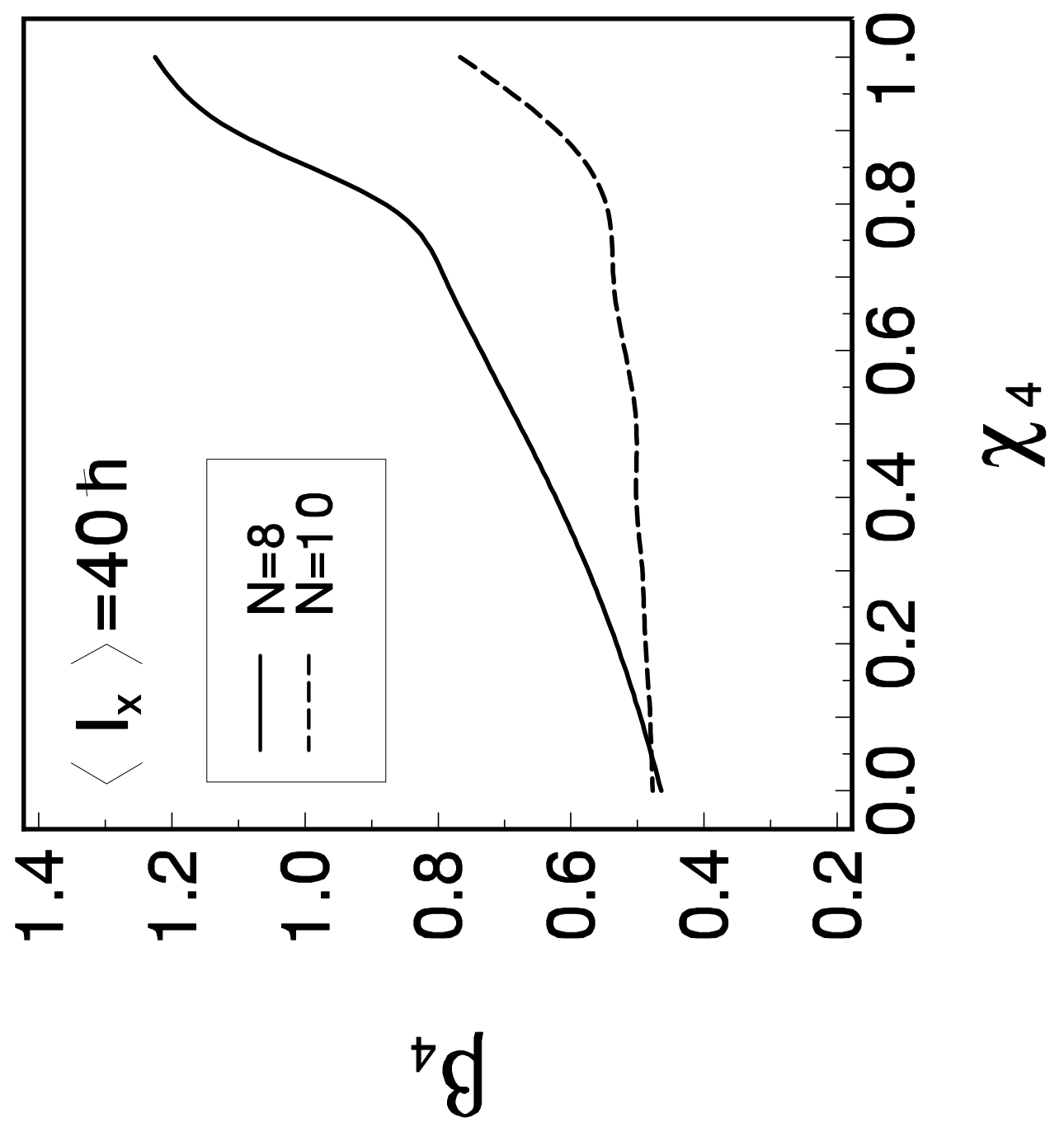

